\title{
Thermal and Electronic Properties of Exfoliated Metal Chalcogenides
}

\author{
Jong-Young Kim, ${ }^{*}$ Soon Mok Choi, ${ }^{\dagger}$ Won-Seon Seo, ${ }^{\dagger}$ and Woo-Seok Cho \\ Icheon Branch, Korea Institute of Ceramic Engineering and Technology, Gyeongchung Rd., Sindun-Myeon, \\ Icheon-si, Kyeonggi-do 467-843, Korea. E-mail: jykim@kicet.re.kr \\ ${ }^{\dagger}$ Green Ceramic Division, Korea Institute of Ceramic Engineering and Technology, 233-5 Gasan-Dong, \\ Guemcheon-Gu, Seoul 153-801, Korea \\ Received July 5, 2010, Accepted September 9, 2010
}

\begin{abstract}
The thermal conductivity of layered metal chalcogenides such as $\mathrm{MT}_{2}(\mathrm{M}=\mathrm{Mo}, \mathrm{W} ; \mathrm{T}=\mathrm{S}, \mathrm{Se})$ shows a marked decrease after exfoliation and subsequent restacking process. Random stacking of two-dimensional crystalline sheets circumvents thermal conduction pathways along a longitudinal direction, which results in a reduction in thermal conductivity. $\mathrm{WS}_{2}$ and $\mathrm{WSe}_{2}$ compounds retain p-type conducting behavior after exfoliation and restacking with decreased electrical conductivity due to the change in carrier concentration. $\mathrm{MoSe}_{2}$ compound exhibits metallic behavior $<130{ }^{\circ} \mathrm{C}$ with a small Seebeck coefficient, which results from metastable 1T-MoSe 2 structure of the restacked phase.
\end{abstract}

Key Words: Thermal properties, Electrical properties

\section{Introduction}

Materials with dense and ordered structures exhibiting low thermal conductivity have attracted much attention due to a variety of potential applications. A number of thermal insulators have been reported; however, these are mostly porous insulators. Electrical and mechanical properties like those of fully dense solids are required in many applications such as thermoelectric device.

Recently, low-dimensional nanostructured materials such as quantum dot superlattices ${ }^{1,2}$ and silicon nanowires ${ }^{3}$ were reported to exhibit reduced thermal conductivity to increase the thermoelectric figure of merit $\left(Z T=S^{2} \sigma T \kappa^{-1}\right.$, where $Z, T, S, \sigma$, and $\kappa$ are a figure of merit, temperature, Seebeck coefficient, electrical conductivity, and thermal conductivity, respectively). ${ }^{1}$ Chiritescu et al. showed that ultralow thermal conductivity in fully dense solids can be achieved in $\mathrm{WSe}_{2}$ films, in which twodimensional crystalline sheets are randomly stacked with turbostratic structure. ${ }^{4}$ Although, in general, insulating amorphous solids and glasses exhibit low thermal conductivity compared to dense solids, low thermal conductivity as small as $0.05 \mathrm{Wm}^{-1} \mathrm{~K}^{-1}$ was observed for turbostratic structured $\mathrm{WSe}_{2}$ film in perpendicular direction to well-crystallized $\mathrm{WSe}_{2}$ sheets. When the layered structure and crystallinity of the film is disrupted by ion radiation, thermal conductivity shows a marked increase, which is contrary to the conception that an amorphous form is better for a thermal insulator.

In this work, we attempted to reduce the thermal conductivity of bulk metal chalcogenides by controlling the disorder of well-crystallized crystalline sheets. We carried out exfoliation through chemical manipulation and obtained a disrupted turbostratic structure, consisting of laterally crystalline sheets, in the $a-b$ plane. The evolution of thermal conductivity, electrical resistivity, and the Seebeck coefficient were discussed with regard to their crystalline structure.

\section{Experimental}

The exfoliated $\mathrm{MT}_{2}(\mathrm{M}=\mathrm{Mo}, \mathrm{W} ; \mathrm{T}=\mathrm{S}, \mathrm{Se})$ compounds were prepared by lithium intercalation and a subsequent exfoliation procedure. ${ }^{5} \mathrm{MT}_{2}$ compounds were purchased from Alfa Aesar $(>99.9 \%)$ and used without further treatment. The pristine compounds were mixed with a 3 molar excess of $n-B u L i$ in hexane and stirred for 3 days. The lithiated powder was washed with hexane thoroughly and exfoliated with $\mathrm{H}_{2} \mathrm{O}$ for 3 days. After exfoliation, the suspension was thoroughly washed with distilled water and centrifuged to get restacked powder. Powder XRD patterns were recorded at room temperature on a Rigaku RINT2000 diffractometer with a $\mathrm{Cu} \mathrm{K} \alpha$ radiation source. X-ray tube voltage and current were at $40 \mathrm{kV}$ and $200 \mathrm{~mA}$, respectively (Figures 1 and 2).

The specimens for transport property measurement were prepared by pressing the restacked polycrystalline powder. The applied pressure in pressing was $1 \mathrm{GPa}$. In order to exclude pressure dependence of conductivity and the intergrain factor, the same pressure is applied to the powder in specimen preparation. Thermal conductivity was determined from thermal diffusivity, $\alpha$, by a laser flash method (Netzsch Instruments, Inc., Burlington, Massachusetts, USA) and heat capacity. ${ }^{6}$ Please refer

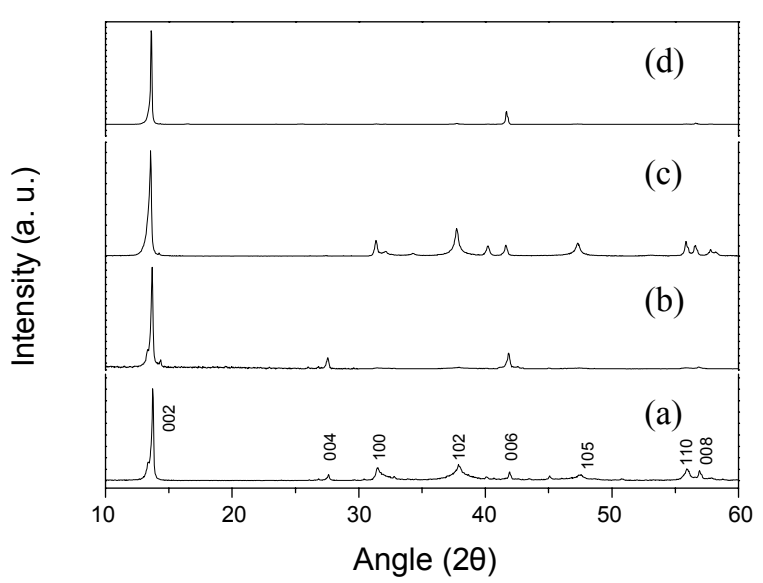

Figure 1. Powder XRD patterns for (a) $\mathrm{MoSe}_{2}$ (pristine), (b) $\mathrm{MoSe}_{2}$ (exfoliated) (c) $\mathrm{WSe}_{2}$ (pristine), and (b) $\mathrm{WSe}_{2}$ (exfoliated) compounds. 


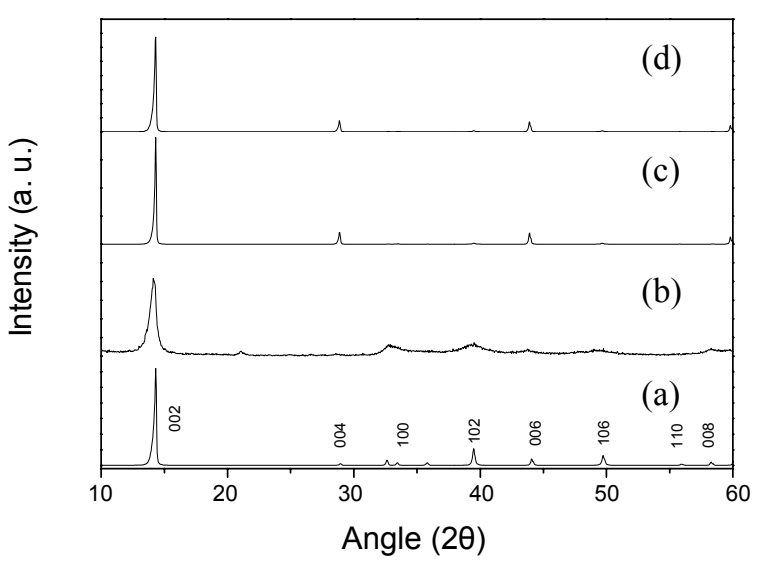

Figure 2. Powder XRD patterns for (a) $\mathrm{MoS}_{2}$ (pristine), (b) $\mathrm{MoS}_{2}$ (exfoliated) (c) $\mathrm{WS}_{2}$ (pristine), and (d) $\mathrm{WS}_{2}$ (exfoliated) compounds.

to supplementary information for details. For Seebeck coefficient, the thermoelectromotive force was measured as a function of the temperature difference using commercial equipment (Ozawa Science, Thermoelectric Property Measurement System, model RZ2001i) on the bar-type sample.

\section{Results and Discussion}

The temperature dependencies of thermal conductivities of the restacked $\mathrm{MT}_{2}(\mathrm{M}=\mathrm{Mo}, \mathrm{W} ; \mathrm{T}=\mathrm{S}, \mathrm{Se})$ are compared with those of pristine $\mathrm{MT}_{2}$ in Figure $3{ }^{7}$ We found out that the thermal conductivities of the restacked samples are significantly lower than those of the pristine samples, mainly because of reduced thermal diffusivity values in the 25 to $300{ }^{\circ} \mathrm{C}$ range. This is because the restacked compounds retain the intralayer crystalline structure; however, the interlayer structure is disrupted during the reaction. In $\mathrm{MT}_{2}$ structure, a hexagonal plane of Mo and $\mathrm{W}$ atoms is bonded to two $\mathrm{S} / \mathrm{Se}$ layers by strong covalentionic bonds, and each two-dimensional $\mathrm{MT}_{2}$ sheet is bonded to adjacent layers by weaker van der Waals forces. These materials can provide several characteristic features such as exfoliation/intercalation, turbostratic disorder due to lattice mismatch, and strain relief due to free slide of the layers.

Chiritescu et al. demonstrated that thermal conductivity of disordered thin films of the layered crystal $\mathrm{WSe}_{2}$ can be as small as $0.05 \mathrm{Wm}^{-1} \mathrm{~K}^{-1}$. Ultralow thermal conductivity was achieved by controlling interlayer structure and thermal pathways in anisotropic materials. The data shown in Figure 3 are consistent with the fact that turbostratic stacking of well-crystallized metal sulfide/selenide sheets circumvents an effective thermal conduction pathway. The stacked layers are substantially randomly arranged about an axis perpendicular to the layer. However, such a dramatic reduction in thermal conductivity as observed in the $\mathrm{WSe}_{2}$ film was not observed in the present system, which might be due to the polycrystalline nature of the samples. The previously reported thermal conductivity values are those of cross-plane conductivity of thin films, which are susceptible to turbostratic disorder of chalcogenide crystals; however, in polycrystalline samples, cross-plane and in-plane terms should be intermixed even though an effect of preferred orientation

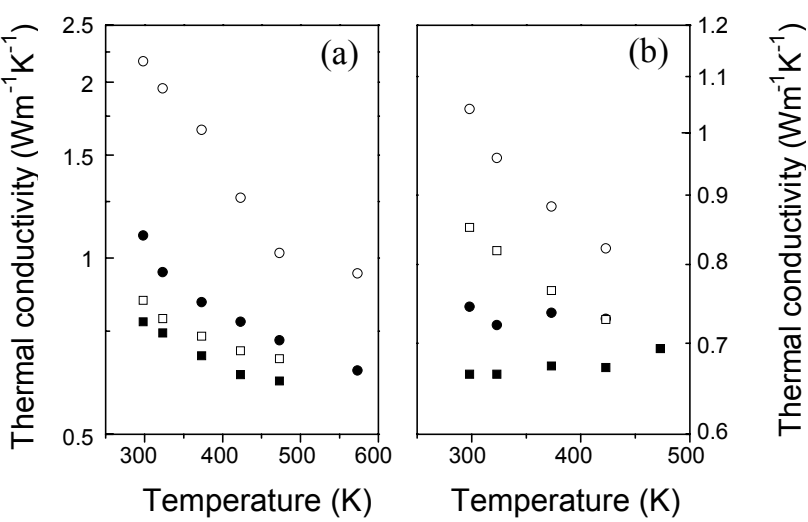

Figure 3. Thermal conductivities of (a) $\mathrm{WSe}_{2}$ (rectangle) and $\mathrm{WS}_{2}$ (circles) and (b) $\mathrm{MoSe}_{2}$ (rectangle) and $\mathrm{MoS}_{2}$ (circles). Solid symbols represent exfoliated and restacked compounds, and open symbols indicate the pristine.

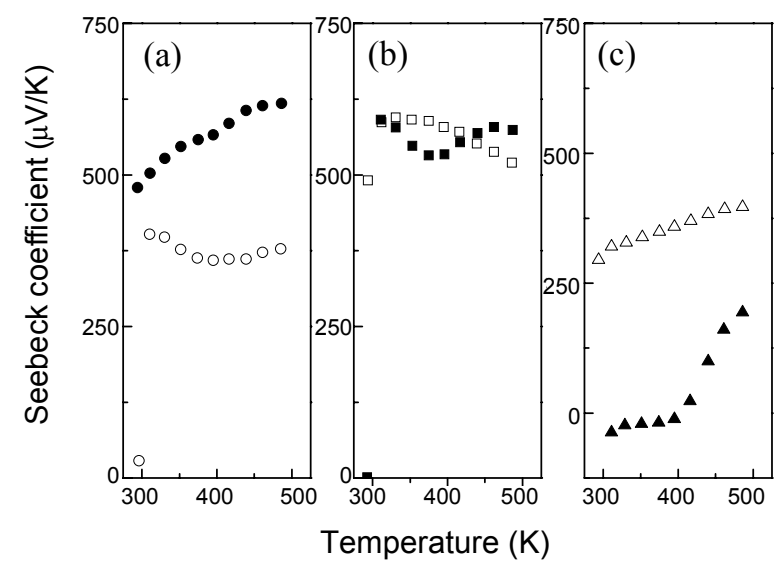

Figure 4. Seebeck coefficients for (a) $\mathrm{WS}_{2}$, (b) $\mathrm{WSe}_{2}$, and (c) $\mathrm{MoSe}_{2}$. Solid symbols represent exfoliated and restacked compounds, and open symbols indicate the pristine.

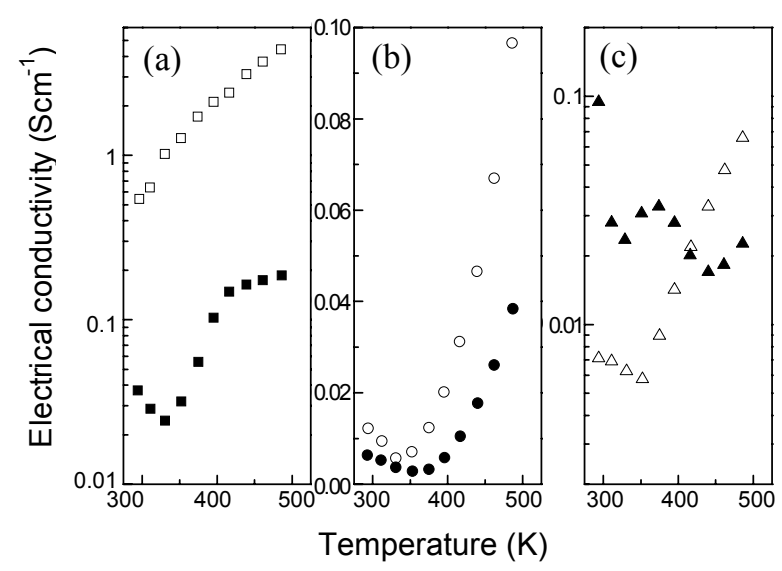

Figure 5. Electrical conductivities for (a) $\mathrm{WS}_{2}$, (b) $\mathrm{WSe}_{2}$, and (c) $\mathrm{MoSe}_{2}$. Solid symbols represent the exfoliated and restacked compounds, and open symbols indicate the pristine.

due to particle shape is involved in specimen preparation.

Figure 4 shows the temperature dependence of the Seebeck coefficient. The $\mathrm{WS}_{2}$ and $\mathrm{WSe}_{2}$ samples show positive values, indicating that $\mathrm{p}$-type conducting behavior is retained during 
the exfoliation and restacking process. The Seebeck coefficient of $\mathrm{WS}_{2}$ increases after exfoliation and restacking, most likely because of the change in carrier concentration. Whereas the Seebeck coefficient of $\mathrm{WS}_{2}$ increases after exfoliation, electrical conductivity decreases with increased carrier concentration, as shown in Figure 4(a). WSe 2 also exhibits semiconducting behavior similar to that of $\mathrm{WS}_{2}$ and comparable Seebeck coefficient after exfoliation with decreased electrical conductivity in the measured temperature range.

However, $\mathrm{MoSe}_{2}$ shows rather complex behavior with a change of sign. As shown in Figure 4(c), the restacked $\mathrm{MoSe}_{2}$ exhibits metallic behavior $<130{ }^{\circ} \mathrm{C}$ with a small Seebeck coefficient value and metal-insulator transition. This metallic conduction in a low temperature range might result from metastable $1 \mathrm{~T}-\mathrm{MoSe}_{2}$ structure of the restacked phase, which is analogous to $\mathrm{MoS}_{2}$ and $\mathrm{WS}_{2}{ }^{8}$ A superstructure at low temperatures induces the distortion of ideal octahedral to a zigzag chain, which results in the splitting of the $d_{x z, y z} / d_{z 2}$ level in the $t_{2 g}$ band. In the case of $\mathrm{MoS}_{2}$ and $\mathrm{WS}_{2}$, irreversible transformation to thermodynamically stable $2 \mathrm{H}-\mathrm{MoS}_{2}$ occurs at rather low temperature $\left(95^{\circ} \mathrm{C}\right.$ for $\left.\mathrm{MoS}_{2}\right){ }^{8}$ therefore, such a transition as that observed in $\mathrm{MoSe}_{2}$ was not observed because of the drying process.

\section{Conclusion}

Thermal conductivity was lowered by in-plane disordered structure due to an exfoliation and restacking process of $\mathrm{MT}_{2}$
$(\mathrm{M}=\mathrm{Mo}, \mathrm{W} ; \mathrm{T}=\mathrm{S}, \mathrm{Se})$. Whereas the Seebeck coefficients exhibit comparable values after the exfoliation and restacking, the electrical conductivities were drastically reduced due to the correlation of carrier concentration and mobility.

Acknowledgment. This research was supported by a grant from the Fundamental R\&D Program for Core Technology of Materials funded by the Ministry of Knowledge Economy, Republic of Korea.

\section{References}

1. Tritt, T. M. Science 1999, 283, 804.

2. Harman, T. C.; Taylor, P. J.; Walsh, M. P.; LaForge, B. E. Science 2002, 297, 2229.

3. Hochbaum, A. I.; Chen, R.; Delgado, R. D.; Liang, W.; Garnett, E. C.; Najarian, M.; Majumdar, A.; Yang, P. Nature 2008, 451, 163.

4. Chiritescu, C.; Cahill, D. G.; Nguyen, N.; Johnson, D.; Bodapati, A.; Keblinski, P.; Zschack, P. Science 2007, 315, 351.

5. Tsai, H.; Heising, J.; Schindler, J. L.; Kannewurf, C. R.; Kanatzidis, M. G. J. Amer. Chem. Soc. 1997, 9, 879.

6. Bolgar, A. S.; Trofimova, Z. A.; Yanaki, A. A. Powder Metall. Met. Ceram. 1990, 29, 382.; Blinder, A. V.; Bolgar, A. S.; Trofimova, Z. A. Powder Metall. Met. Ceram. 1993, 32, 234.

7. Reliable electrical conductivity and Seebeck coefficient for $\mathrm{MoS}_{2}$ cannot be obtained because of the contamination of platinum wire due to the evaporation above $100{ }^{\circ} \mathrm{C}$.

8. Dungey, K. E.; Curtis, M. D.; Penner-Hahn, J. E. Chem. Mater. 1998, 10, 2152; Heising, J.; Kanatzidis, M. G. J. Amer. Chem. Soc. 1999, 121, 11720. 